\title{
Thin-slab maximum intensity projection of CT angiography for collateral score and clot burden score evaluation: comparison with conventional CT angiography
}

\author{
Li-Ming Wei, Yue-Qi Zhu, Hai-Tao Lu, Jun-Gong Zhao \\ Department of Diagnostic and Interventional Radiology, Shanghai Jiao Tong University Affiliated Sixth People's Hospital, Shanghai, China
}

Contributions: (I) Conception and design: JG Zhao; (II) Administrative support: LM Wei; (III) Provision of study materials or patients: LM Wei; (IV) Collection and assembly of data: YQ Zhu; (V) Data analysis and interpretation: LM Wei, HT Lu; (VI) Manuscript writing: All authors; (VII) Final approval of manuscript: All authors.

Correspondence to: Jun-Gong Zhao, MD. No. 600, Yi Shan Road, Shanghai 200233, China. Email: zhaojungongradio@hotmail.com.

\begin{abstract}
Background: This study aimed to exam the effects of thin-slab maximum intensity projection (TS-MIP) of computed tomography angiography (CTA) for collateral score (CS) and clot burden score (CBS) evaluation in patients with large-vessel-occlusion (LVO) stroke in the anterior circulation.

Methods: Of 241 consecutive patients with LVO stroke admitted to our center between August 2015 and June 2020, 187 patients were enrolled. CS and CBS were evaluated on conventional CTA and TS-MIP separately. Outcome at 90 days was classified as good if modified Rankin scale (mRS) was $\leq 2$ and as poor if mRS was $>2$. The correlations between CS and CBS and clinical outcomes were assessed. Receiver operating characteristic (ROC) curve analysis was used to determine the diagnostic values of CS and CBS. Multivariate logistic regression analysis was performed to identify the independent predictors of 90-day good clinical outcomes.
\end{abstract}

Results: The correlation coefficient for clinical outcomes was significantly better for CS based on TS-MIP than that based on conventional CTA $(-0.444$ vs. $-0.285, \mathrm{P}=0.039)$; no significant difference was found in the CBS evaluation (TS-MIP: -0.356 vs. conventional CTA: $-0.320, \mathrm{P}=0.348$ ). For predicting good clinical outcomes, TS-MIP-based CS was associated with larger area under the curve (AUC) (0.709 vs. 0.609, $\mathrm{P}=0.004)$ and higher sensitivity $(69.1 \%$ vs. $42.0 \%, \mathrm{P}=0.001)$ than CS based on CTA. In multivariable logistic regression analysis, the factors independently associated with good outcomes were National Institutes of Health Stroke Scale (NIHSS) score at admission (OR =1.147; $\mathrm{P}<0.001)$, TS-MIP-based CS (OR =0.326; $\mathrm{P}<0.001$ ), final modified treatment in cerebral infarction (mTICI) score of $2 \mathrm{~b} / 3$ (OR $=0.098 ; \mathrm{P}<0.001$ ), and hemorrhagic transformation $(\mathrm{OR}=3.662 ; \mathrm{P}<0.001)$.

Conclusions: TS-MIP-CTA is superior to conventional CTA for evaluation CS and CBS, and TS-MIPbased CS may be a useful predictor of clinical outcome.

Keywords: Maximum intensity projection; collateral score; clot burden score; stroke

Submitted Feb 09, 2021. Accepted for publication Sep 03, 2021.

doi: 10.21037/qims-21-170

View this article at: https://dx.doi.org/10.21037/qims-21-170

\section{Introduction}

Recent trials have shown that timely and successful recanalization through mechanical thrombectomy (MT) can rescue at-risk tissue and reverse clinical impairment after acute large-artery-occlusion (LVO) in the anterior circulation (1-6). Nevertheless, the final prognosis depends on many factors besides recanalization of the 
primary occlusive arterial lesion. Collateral integrity and clot extent are important determinants of clinical and radiologic outcomes and have been used as predictors of recovery. Good collaterals increase probability of successful recanalization and decrease risk of hemorrhagic transformation following MT $(7,8)$; meanwhile, high clot burden suggests proximal occlusion, more severe clinical deficits, and a less favorable 3-month clinical outcome (9). Thus, acute and fast evaluation of collateral score (CS) and clot burden score (CBS) before MT can help guide treatment decisions and predict outcomes. In most studies to date, CBS and CS evaluation studies have been conducted based on conventional CT angioplasty (CTA) techniques, including three-dimensional volume rendering (VR) and full-slab maximum intensity projection (FSMIP) reconstructions $(8,10)$. However, as some authors have pointed out, thin-slab MIP (TS-MIP) for CTA, in this technique, MIP images at a small section thickness are acquired to improve both noise and coplanar effects. It also affords the option to interactively scroll through datasets on a picture archiving and communication system (PACS) workstation $(11,12)$. Thus, TS-MIP might be superior to conventional CTA for evaluation of CS and CBS because it can better define the complex vascular anatomy, and allow the visualization of small intraparenchymal branches.

In this study, we aimed to compare the predictive values of CS and CBS evaluated on TS-MIP of CTA to versus conventional CTA for clinical outcomes in patients with acute LVO stroke in the anterior circulation.

\section{Methods}

\section{Patients' information}

The study was conducted in accordance with the Declaration of Helsinki (as revised in 2013), and was approved by the ethics committees of Shanghai Sixth People's hospital. Individual consent for this retrospective analysis was waived. The study sample was selected from among 241 consecutive patients with acute LVO stroke who were admitted to our center between August 2015 and June 2020. Patients were eligible for inclusion in this study if: (I) they had unilateral LVO in the internal carotid artery (ICA) or middle carotid artery (MCA; M1 or proximal M2) as determined using head CTA performed on admission, and (II) they had been treated with MT. The exclusion criteria were as follows: MT not performed (13 patients), a poor CTA image quality unsuitable for further analysis (10 patients), and stroke due to LVO in the posterior circulation
(31 patients). Functional outcomes were assessed using the modified Rankin scale (mRS) scores at 90 days after MT. A mRS score of 0-2 was defined as a good clinical outcome, while $\mathrm{mRS}>2$ indicated a poor outcome.

\section{CTA protocol}

CTA was performed on a 64-MDCT scanner (Brilliance 64; Philips Healthcare; Best, the Netherlands) with the following parameters: tube voltage, $120 \mathrm{kV}$; tube current, $435 \mathrm{~mA}$; and rotation speed, $0.75 \mathrm{~s}$. An IV contrast agent $(120 \mathrm{~mL}$ of Ultravist, Bayer Vital GmbH; iodine concentration, $300 \mathrm{mg} / \mathrm{mL}$ ) was administered at a flow rate of $4 \mathrm{~mL} / \mathrm{s}$. Scanning was started when the CT attenuation in the ascending aorta was $120 \mathrm{HU}$. CTA images were reconstructed at a thickness of $0.67 \mathrm{~mm}$. VR and conventional MIP post-processing were performed on a multimodality workstation (Philips Medical Systems, Philips Healthcare, Best, The Netherlands) for each patient. For TS-MIP construction, the acquired $0.67-\mathrm{mm}$ axial-slice CTA images were transferred to a dedicated workstation (SyngoXWP; Siemens Healthcare, Erlangen, Germany), and loaded into the InSpace section at the workstation; the DSA Layout application was used to reconstruct coronal MIP images with $10-\mathrm{mm}$ slice thickness.

\section{CS and CBS scoring}

Two neuroradiologists (Y.Q.Z. and J.G.Z., 12 and 22 years of experience in interventional neuroradiology, respectively) who were blinded to the clinical data independently estimated the CBS and CS on conventional CTA and TSMIP, separately. If there was a disagreement between the two readers, a decision was reached by consensus. Collaterals were graded using the system described by Miteff et al. (13), as grade 1 (or poor), i.e., reconstitution of only distal superficial MCA branches; grade 2 (or moderate), i.e., reconstitution of some MCA branches in the sylvian fissure; or grade 3 (or good), i.e., reconstitution of major MCA branches distal to the occlusion. The CBS, which defines the extent of the thrombus in the proximal anterior circulation by location, is scored on a scale of $0-10$ (9), with 0 implying complete multisegment vessel occlusion and 10 implying absence of clot. During scoring, 2 points each are subtracted for the thrombus in the supraclinoid ICAs, proximal half of the MCA trunk, and distal half of the MCA trunk, and 1 point each is subtracted for the thrombus in the infraclinoid ICA and anterior cerebral artery (ACA) and M2 branches. 


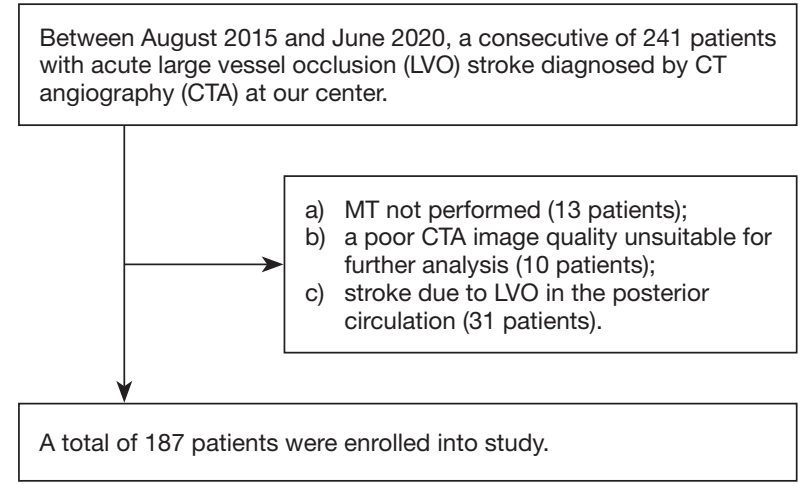

Figure 1 Flow diagram of the protocol for inclusion of patients in the study.

The thrombus may be partially or completely occlusive.

\section{Statistical analysis}

Continuous variables were summarized as means \pm standard deviation or as medians [with interquartile range (IQR)], depending on the normality of distribution. The Student t-test was used for normally distributed data, while the Mann-Whitney $U$ test was used for data that were not normally distributed. The $\chi^{2}$ test was applied for categorical variables. Clinical characteristics, risk factors, imaging features, and clinical outcomes were compared. Receiver operating characteristic (ROC) curve and multivariate logistic regression analyses were performed for the CBS and CS to predict clinical outcomes. The area under the curve (AUC), sensitivity, specificity, positive predictive value (PPV), and negative predictive value (NPV) were calculated, and thresholds were determined. The kappa statistic was used to assess inter-reader agreement in evaluation of CBS and CS on TS-MIP and conventional CTA; agreement was graded as poor $(\kappa \leq 0.20)$ fair $(\kappa=0.21-0.40)$, moderate $(\kappa=0.41-0.60), \operatorname{good}(\kappa=0.61-0.80)$, or very $\operatorname{good}(\kappa=$ 0.81-1.0). Statistical analyses were performed using SPSS version 20.0 (IBM Corp., Armonk, NY, USA). Statistical significance was set at $\mathrm{P}<0.05$.

\section{Results}

\section{Baseline data of all patients}

A total of 187 patients (111 males, 76 females; mean age $70.7 \pm 11.7$ years) were included in this study. The flowchart in Figure 1 outlines the process used for inclusion and exclusion of patients. Baseline median admission National Institutes of Health Stroke Scale (NIHSS) score was 14 (IQR, 10-18). Mean admission systolic blood pressure was $145.5 \pm 19.5 \mathrm{mmHg}$. Intravenous thrombolysis was administered to 57 (30.5\%) patients. Median CBS was 6 (IQR, 4-8) on both conventional CTA and TS-MIP; while median CS was 2 (IQR, 1-3) on conventional CTA and 2 (IQR, 2-3) on TS-MIP. Final modified treatment in cerebral infarction (mTICI) score of $2 \mathrm{~b}$ or 3 was achieved in $159(85.0 \%)$ patients. Ten $(5.3 \%)$ patients receiving thrombolysis were recanalized. Good clinical outcome (mRS $\leq 2$ at 90 days) was achieved in $81(43.3 \%)$ patients.

\section{CBS and CS associated with outcomes}

TS-MIP-based CS showed significantly stronger correlation with clinical outcome than conventional CTA-based CS (0.444 vs. $0.285, \mathrm{P}=0.039)$. However, the correlation of TS-MIP-based CBS and conventional CTA-based with clinical outcome was not significantly different $(0.356$ vs. 0.320, $\mathrm{P}=0.348$ ) (Figures 2,3). For prediction of good clinical outcomes in ROC analysis, CS based on TS-MIP showed a larger AUC than CS based on conventional CTA (0.709 vs. $0.609, \mathrm{P}=0.004)$, and no significant difference was observed between CBS based on TS-MIP and CBS based on conventional CTA (0.678 vs. $0.658, \mathrm{P}=0.307$ ) (Figure 4). The sensitivity, specificity, PPV and NPV of CS based on TSMIP for predicting good clinical outcome were $69.1 \%(95 \%$ CI: $57.8-78.7 \%$ ), $66.0 \%$ (95\% CI: $56.1-74.8 \%$ ), $60.9 \%$ (95\% CI: 50.1-70.7\%), $73.7 \%$ (95\% CI: 63.5-81.9\%), while the corresponding values for CS based on conventional CTA were $42.0 \%$ (95\% CI: 31.3-53.5\%), 80.2\% (95\% CI: $71.1-87.1 \%$ ), $61.8 \%$ (95\% CI: $47.7-74.3 \%$ ) and $64.4 \%$ (95\% CI: 55.5-72.4\%), respectively, which indicated that CS based on TS-MIP had higher sensitivity $(\mathrm{P}=0.001)$ and lower specificity $(\mathrm{P}=0.020)$ than $\mathrm{CS}$ based on conventional CTA.

\section{Factors associated with clinical outcomes}

The clinical characteristics of patients with good and poor clinical outcomes are shown in Table 1. Patients with good clinical outcomes had significantly lower admission NIHSS score [10 (IQR, 6-15) vs. 16 (IQR, 12.8-20), $\mathrm{P}<0.001$ ], greater rate of mTICI scores of $2 \mathrm{~b}$ or $3(95.1 \%$ vs. $77.4 \%$, $\mathrm{P}=0.001$ ), and lower hemorrhagic transformation rate (9.9\% vs. $31.1 \%, \mathrm{P}<0.001)$ than patients with poor clinical outcomes. The proportion of ICA-involved LVO was significantly greater in patients with poor clinical outcomes 

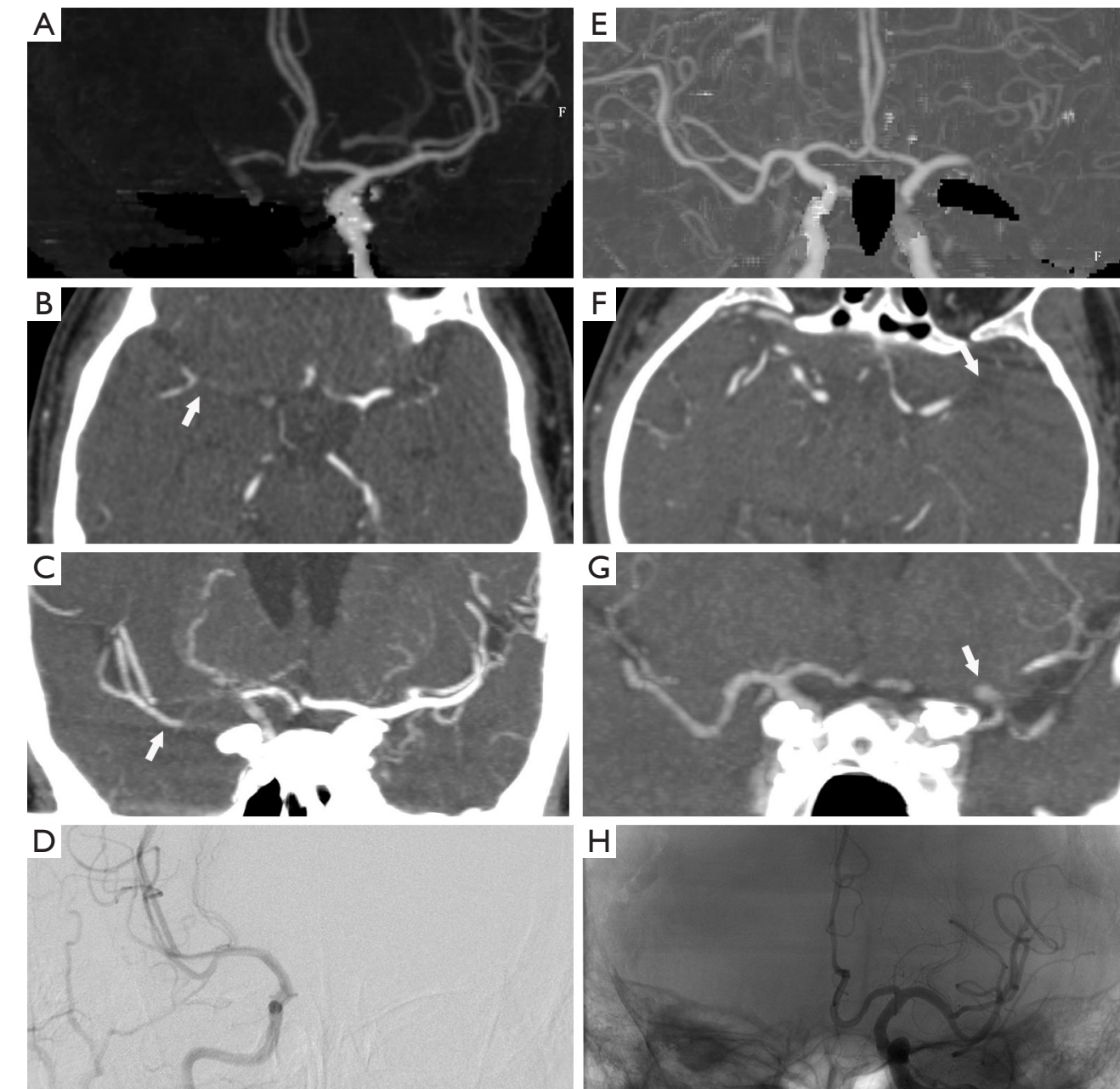

Figure 2 Illustrative case of collateral status (CS) based on CT angiography (CTA). (A-D) A 74-year-old man with left hemiparesis and impaired consciousness due to right internal carotid artery large vessel occlusion (ICA-LVO) stroke: (A,B) conventional CTA showed moderate CS because the middle cerebral artery (MCA) branches were reconstituted within the Sylvian fissure (arrow); (C) however, thinslab maximum intensity projection (TS-MIP) showed good CS because the proximal M2 segment was reconstituted at the distal end of the occlusion vessel; (D) digital subtraction angiography (DSA) shows that the right ICA was recanalized, and the patient had a 90-day mRS of 1. (E-H) A 66-year-old man with right hemiparesis due to left MCA-LVO stroke: (E,F) conventional CTA showed poor CS because only the distal superficial MCA branches were reconstituted (arrow); (G) however, TS-MIP showed good CS because the distal M1 segment was reconstituted at the distal end of the occlusion vessel; $(\mathrm{H})$ DSA shows that the left MCA was recanalized, and the patient had a 90 -day mRS of 2.

than in patients with good clinical outcomes $(40.6 \%$ vs. $27.2 \%, \mathrm{P}=0.002$ ).

Patients with good clinical outcomes had significantly higher CBS [6 (IQR, 6-9) vs. 6 (IQR, 1-7), $\mathrm{P}<0.001$ based on conventional CTA; 7 (IQR, 6-9) vs. 6 (IQR, 2.8-8), $\mathrm{P}<0.001$ based on TS-MIP] and CS [2 (IQR, 1-3) vs. 1 (IQR, 1-2), $\mathrm{P}=0.006$ based on conventional CTA; 3 (IQR, 2-3) vs. 2 (IQR, 1-3), $\mathrm{P}<0.001$ based on TS-MIP] than patients with poor clinical outcomes. Multivariable logistic regression analysis revealed that the factors independently associated with good clinical outcome were admission NHISS score $(\mathrm{OR}=1.147$; 95\% CI: 1.075-1.223; $\mathrm{P}<0.001)$, CS based on TS-MIP (OR =0.326; 95\% CI: 0.192-0.553; $\mathrm{P}<0.001$ ), final mTICI score of $2 \mathrm{~b}$ or 3 (OR $=0.098 ; 95 \%$ CI: $0.028-0.343 ; \mathrm{P}<0.001)$, and hemorrhagic transformation $(\mathrm{OR}=3.662 ; 95 \% \mathrm{CI}: 1.400-9.578 ; \mathrm{P}<0.001)$ (Table 2).

\section{Inter-reader agreement}

The correlation coefficient for CBS indicated good 

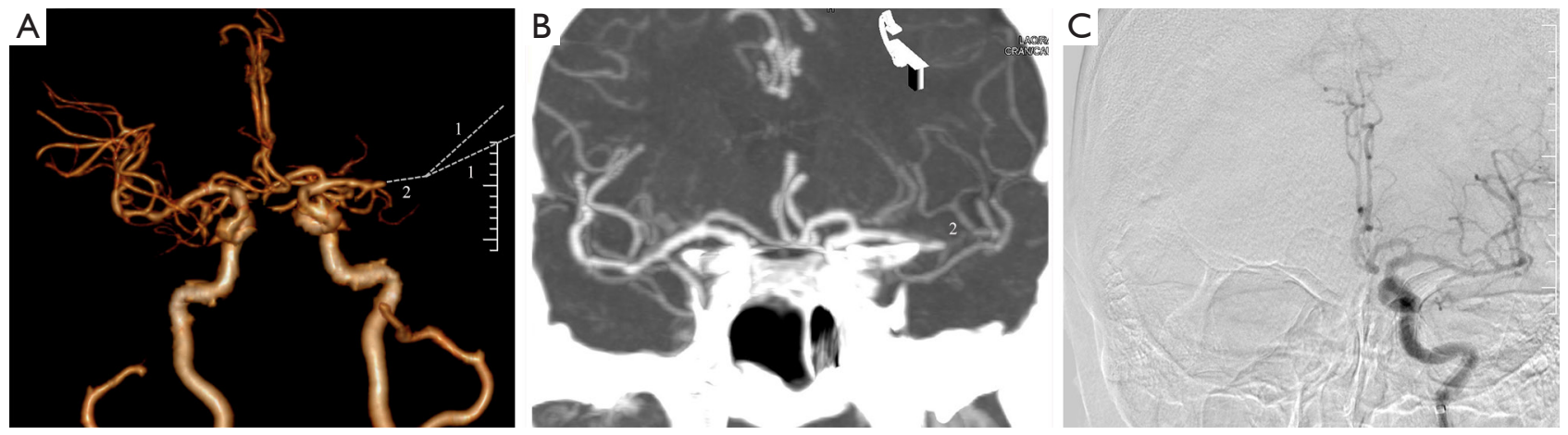

Figure 3 Illustrative case of clot burden score (CBS) based on CT angiography (CTA). (A) On the basis of conventional CTA, a CBS of 6 was assigned to a patient with left MCA-LVO; (B) however, on the basis of TS-MIP, a CBS of 8 was assigned; (C) DSA shows that the left MCA was recanalized, and the patient had a 90-day mRS score of 0.
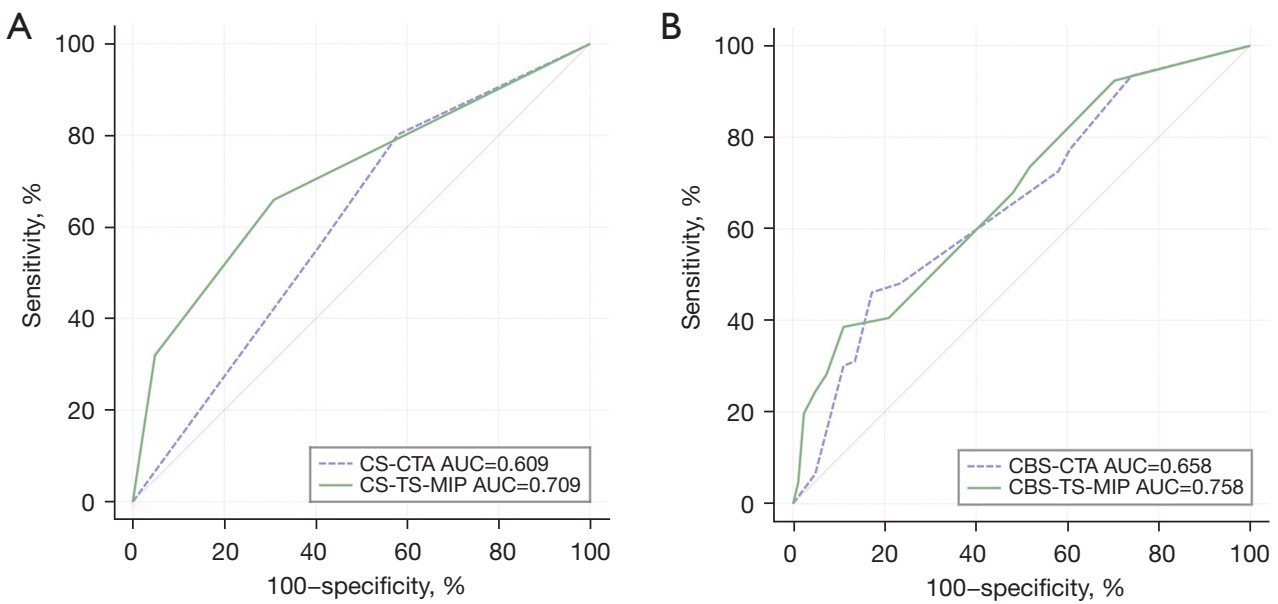

Figure 4 Comparation of Receiver operating characteristic (ROC) analyse for collateral status (CS) and clot burden score (CBS) prediction of good clinical outcome [modified Rankin scale (mRS) $\leq 2$ ].

agreement between TS-MIP and conventional CTA $(\mathrm{R}=0.898)$. However, the correlation coefficient for CS indicated fair agreement between TS-MIP and conventional CTA ( $\mathrm{R}=0.617)$. Good interobserver correlation for CBS $(\mathrm{R}=0.971)$ and $\mathrm{CS}(\mathrm{R}=0.871)$ was achieved between the two readers.

\section{Discussion}

To our knowledge, this is the first study to demonstrate the value and application of TS-MIP of CTA for evaluating CS and CBS in patients with acute LVO stroke. The study showed that CS and CBS estimated on TS-MIP were more closely associated with outcome than CS and CBS estimated on conventional CTA. Further, for prediction of clinical outcome, TS-MIP-based CS had greater predictive value and higher sensitivity than conventional CTA-based CS.

Collateral supply is very important in the disease development of cerebral ischemia in acute stroke, as it is associated with clinical outcome, infarct volume, hemorrhage transformation risk, and recanalization rates (14). However, it is difficult to quantify collateral supply due to its diminutive and complex routes. As we all know, CTA can be used to screen acute ischemic stroke for determining the occlusion site (15), and it can also provide a simple and reproducible assessment method for collateral supply evaluation to confirm the association between collateral supply and clinical outcome. Up to now, several CTAbased collateral grading systems have been proposed for patients with acute LVO strokes, including the ASITN/SIR, 
Table 1 Patient with good clinical outcomes ( $\mathrm{mRS}$ score $\leq 2$ ) and poor clinical outcome (mRS score, $>2$ ) at 90 days

\begin{tabular}{|c|c|c|c|c|}
\hline & Total $(n=187)$ & mRS score $\leq 2(n=81)$ & mRS score $>2(n=106)$ & $P$ value \\
\hline Male sex, n (\%) & $111(59.4)$ & $49(60.5)$ & $62(58.5)$ & 0.782 \\
\hline Median admission NHISS [IQR] & $14[10-18]$ & $10[6-15]$ & 16 [12.8-20] & $<0.001^{*}$ \\
\hline Mean admission systolic pressure $(\mathrm{mmHg})$ & $145.5 \pm 19.5$ & $145.0 \pm 18.3$ & $145.8 \pm 20.5$ & 0.795 \\
\hline Hypertension & $123(65.8)$ & $49(60.5)$ & $74(69.8)$ & 0.183 \\
\hline Hyperlipidemia & $21(11.2)$ & $10(12.3)$ & $11(10.4)$ & 0.673 \\
\hline Diabetes & $46(24.6)$ & $15(18.5)$ & $31(29.2)$ & 0.091 \\
\hline Cardioembolic & $97(51.9)$ & $38(46.9)$ & $59(55.7)$ & \\
\hline Stroke of other determined cause & $3(1.6)$ & $1(1.2)$ & $2(1.9)$ & \\
\hline Cryptogenic & $49(26.2)$ & $26(32.1)$ & $23(21.7)$ & \\
\hline HAS, n (\%) & $92(49.2)$ & $38(46.9)$ & $54(50.9)$ & 0.585 \\
\hline Site of vessel occlusion, n (\%) & & & & $0.002^{*}$ \\
\hline Internal carotid artery & $65(34.8)$ & $22(27.2)$ & $43(40.6)$ & \\
\hline \multicolumn{5}{|l|}{ Middle cerebral artery } \\
\hline First segment & $93(49.7)$ & $38(46.9)$ & $55(51.9)$ & \\
\hline \multicolumn{5}{|l|}{ CS, median [IQR] } \\
\hline Based on CTA & $2[1-3]$ & $2[1-3]$ & $1[1-2]$ & $0.006^{\star}$ \\
\hline Good & $55(29.4)$ & $34(42.0)$ & $21(19.8)$ & 0.004 \\
\hline Moderate & $40(21.4)$ & $14(17.3)$ & $26(24.5)$ & \\
\hline Poor & $92(49.2)$ & $33(40.7)$ & $59(55.7)$ & \\
\hline Based on TS-MIP & $2[2-3]$ & $3[2-3]$ & $2[1-3]$ & $<0.001^{\star}$ \\
\hline Good & $92(49.2)$ & $56(69.1)$ & $36(34.0)$ & $<0.001^{\star}$ \\
\hline Moderate & $57(30.5)$ & $21(25.9)$ & $36(34.0)$ & \\
\hline Poor & $38(20.3)$ & $4(4.9)$ & $34(32.1)$ & \\
\hline Final score on $\mathrm{mTICI} 2 \mathrm{~b}$ or $3, \mathrm{n}(\%)$ & $159(85.0)$ & $77(95.1)$ & $82(77.4)$ & 0.001 \\
\hline Hemorrhagic transformation & $41(21.9)$ & $8(9.9)$ & $33(31.1)$ & $<0.001^{*}$ \\
\hline
\end{tabular}

* indicates a significant difference. mRS, modified Rankin scale; NIHSS, National Institutes of Health Stroke Scale; HAS, Hyper-dense artery sign; IQR, Interquartile range; CBS, Clot burden score; CS, Collateral score; CTA, CT angiography; TS-MIP, Thin-slab maximum intensity projection; $\mathrm{mTICl}$, modified thrombolysis in cerebral infarction. 
Table 2 Multivariate logistic regression analysis of good clinical outcome (mRS score $\leq 2$ at 90 days)

\begin{tabular}{|c|c|c|c|c|}
\hline Variables & $P$ value & OR & Lower $\mathrm{Cl}$ of $\mathrm{OR}$ & Upper $\mathrm{Cl}$ of OR \\
\hline CS based on TS-MIP & $<0.001$ & 0.326 & 0.192 & 0.553 \\
\hline Final score on $\mathrm{mTICI} 2 \mathrm{~b}$ or 3 & $<0.001$ & 0.098 & 0.028 & 0.343 \\
\hline Hemorrhagic transformation & 0.008 & 3.662 & 1.400 & 9.578 \\
\hline
\end{tabular}

mRS, modified Rankin scale; OR, Odds ratio; Cl, Confidence interval; NIHSS, National Institutes of Health Stroke Scale; CS, Collateral score; TS-MIP, Thin-slab maximum intensity projection; $\mathrm{mTICI}$, modified thrombolysis in cerebral infarction.

ASPECTS, Christoforidis and Miteff collateral grading systems (13,16-18), however, there is no consensus on which one is the best. We chose the Miteff et al. grading system in this study because it can reliably identify both favorable and poor outcomes in thrombolyzed acute anterior circulation ischemic stroke, whereas the other grading systems can only predict poor outcomes (19).

MIP images are multiplanar slab images in which only the highest attenuation value of the data in a volume of interest is used to generate a single two-dimensional image and projects for better delineation of the regional anatomy and a better understanding of the disease process (20). Moreover, the sliding thin slab method can offer better results with MIP images since it acquires less contiguous slabs than conventional MIP to improve the conspicuity of small areas of interest $(11,12)$. Therefore, we used TS-MIP images to visualize small intraparenchymal branches for evaluation of collateral vessels in this study and found that both TS-MIP based CS and CBS showed higher correlation coefficients than those based on conventional CTA $(0.356$ vs. 0.320 and $0.444 v s .0 .356$ ) for the correlation with good clinical outcomes, indicating that TS-MIP was more reliable than conventional CTA in evaluating CS and CBS. Additionally, in comparison with CS based on CTA, CS based on TS-MIP showed a larger AUC for good clinical outcomes (0.709 vs. 0.609, $\mathrm{P}=0.004)$ with higher sensitivity (69.1\% vs. $42.0 \%, \mathrm{P}<0.001)$, although no significant difference was observed between CBS based on TS-MIP and CBS based on CTA. Multivariable logistic regression analysis revealed that CS based on TS-MIP, not CS based on CTA, was associated with good clinical outcomes. These findings highlight the value of the TS-MIP technique for $\mathrm{CS}$ and CBS evaluation.

Some limitations of this study must be acknowledged. First, the correlation of CS and CBS and radiologic outcomes were not evaluated, such as ischemic core and ischemic penumbra, since related software was not available at our institution during this period, and it needs to be further studied. Second, as a retrospective study, there is inevitably the potential for selection and information bias; further randomized controlled trials are needed to exclude these biases. Third, this is a study with small sample size at a single center; additional multicenter clinical experience with a larger number of patients will be needed to confirm the value TS-MIP for evaluation CS and CBS.

\section{Conclusions}

The TS-MIP technique appears to be superior to conventional CTA techniques for evaluation of CS and CBS in patients with stroke due to LVO in the anterior circulation. TS-MIP-based CS evaluation may help predict clinical outcome in patients with acute stroke. Whether it could facilitate the choice of route treatment remains to be determined by meta-analyses of existing trials and prospective registries performed in this field.

\section{Acknowledgments}

Funding: This work was supported by the National Natural Science Foundation of China (Nos. 81901845, 81671791), Science Foundation of Shanghai Jiaotong University Affiliated Sixth People's Hospital (No. 201818), and Shanghai key discipline of medical imaging (No: 2017ZZ02005).

\section{Footnote}

Conflicts of Interest: All authors have completed the ICMJE uniform disclosure form (available at https://dx.doi. org/10.21037/qims-21-170). The authors have no conflict of interests to declare.

Ethical Statement: The authors are accountable for all 
aspects of the work in ensuring that questions related to the accuracy or integrity of any part of the work are appropriately investigated and resolved. The study was conducted in accordance with the Declaration of Helsinki (as revised in 2013), and was approved by the ethics committee of Shanghai Sixth People's Hospital. Individual consent for this retrospective analysis was waived.

Open Access Statement: This is an Open Access article distributed in accordance with the Creative Commons Attribution-NonCommercial-NoDerivs 4.0 International License (CC BY-NC-ND 4.0), which permits the noncommercial replication and distribution of the article with the strict proviso that no changes or edits are made and the original work is properly cited (including links to both the formal publication through the relevant DOI and the license). See: https://creativecommons.org/licenses/by-nc-nd/4.0/.

\section{References}

1. Berkhemer OA, Fransen PS, Beumer D, van den Berg LA, Lingsma HF, Yoo AJ, et al. A randomized trial of intraarterial treatment for acute ischemic stroke. $\mathrm{N} \mathrm{Engl} \mathrm{J}$ Med 2015;372:11-20.

2. Campbell BC, Mitchell PJ, Kleinig TJ, Dewey HM, Churilov L, Yassi N, et al. Endovascular therapy for ischemic stroke with perfusion-imaging selection. $\mathrm{N} \mathrm{Engl}$ J Med 2015;372:1009-18.

3. Goyal M, Demchuk AM, Menon BK, Eesa M, Rempel JL, Thornton J, et al. Randomized assessment of rapid endovascular treatment of ischemic stroke. N Engl J Med 2015;372:1019-30.

4. Saver JL, Goyal M, Bonafe A, Diener HC, Levy EI, Pereira VM, et al. Stent-retriever thrombectomy after intravenous t-PA vs. t-PA alone in stroke. $\mathrm{N}$ Engl J Med 2015;372:2285-95.

5. Jovin TG, Chamorro A, Cobo E, de Miquel MA, Molina CA, Rovira A, et al. Thrombectomy within 8 hours after symptom onset in ischemic stroke. N Engl J Med 2015;372:2296-306.

6. Nogueira RG, Jadhav AP, Haussen DC, Bonafe A, Budzik RF, Bhuva P, et al. Thrombectomy 6 to 24 Hours after Stroke with a Mismatch between Deficit and Infarct. N Engl J Med 2018;378:11-21.

7. Berkhemer OA, Jansen IG, Beumer D, Fransen PS, van den Berg LA, Yoo AJ, et al. Collateral Status on Baseline Computed Tomographic Angiography and Intra-Arterial Treatment Effect in Patients With Proximal Anterior
Circulation Stroke. Stroke 2016;47:768-76.

8. Al-Dasuqi K, Payabvash S, Torres-Flores GA, Strander SM, Nguyen CK, Peshwe KU, Kodali S, Silverman A, Malhotra A, Johnson MH, Matouk CC, Schindler JL, Sansing LH, Falcone GJ, Sheth KN, Petersen NH. Effects of Collateral Status on Infarct Distribution Following Endovascular Therapy in Large Vessel Occlusion Stroke. Stroke 2020;51:e193-202.

9. Puetz V, Dzialowski I, Hill MD, Subramaniam S, Sylaja PN, Krol A, O'Reilly C, Hudon ME, Hu WY, Coutts SB, Barber PA, Watson T, Roy J, Demchuk AM; Calgary CTA Study Group. Intracranial thrombus extent predicts clinical outcome, final infarct size and hemorrhagic transformation in ischemic stroke: the clot burden score. Int J Stroke 2008;3:230-6.

10. Tan IY, Demchuk AM, Hopyan J, Zhang L, Gladstone D, Wong K, Martin M, Symons SP, Fox AJ, Aviv RI. $\mathrm{CT}$ angiography clot burden score and collateral score: correlation with clinical and radiologic outcomes in acute middle cerebral artery infarct. AJNR Am J Neuroradiol 2009;30:525-31.

11. Kawel N, Seifert B, Luetolf M, Boehm T. Effect of slab thickness on the CT detection of pulmonary nodules: use of sliding thin-slab maximum intensity projection and volume rendering. AJR Am J Roentgenol 2009;192:1324-9.

12. Ertl-Wagner BB, Bruening R, Blume J, Hoffmann RT, Mueller-Schunk S, Snyder B, Reiser MF. Relative value of sliding-thin-slab multiplanar reformations and sliding-thin-slab maximum intensity projections as reformatting techniques in multisection CT angiography of the cervicocranial vessels. AJNR Am J Neuroradiol 2006;27:107-13.

13. Miteff F, Levi CR, Bateman GA, Spratt N, McElduff $\mathrm{P}$, Parsons MW. The independent predictive utility of computed tomography angiographic collateral status in acute ischaemic stroke. Brain 2009;132:2231-8.

14. Liebeskind DS, Tomsick TA, Foster LD, Yeatts SD, Carrozzella J, Demchuk AM, Jovin TG, Khatri P, von Kummer R, Sugg RM, Zaidat OO, Hussain SI, Goyal M, Menon BK, Al Ali F, Yan B, Palesch YY, Broderick JP; IMS III Investigators. Collaterals at angiography and outcomes in the Interventional Management of Stroke (IMS) III trial. Stroke 2014;45:759-64.

15. Kremenova K, Holesta M, Peisker T, Girsa D, Weichet J, Lukavsky J, Malikova H. Is limited-coverage CT perfusion helpful in treatment decision-making in patients with acute ischemic stroke? Quant Imaging Med 
Surg 2020;10:1908-16.

16. Higashida RT, Furlan AJ, Roberts H, Tomsick T, Connors B, Barr J, Dillon W, Warach S, Broderick J, Tilley B, Sacks D; Technology Assessment Committee of the Society of Interventional Radiology. Trial design and reporting standards for intra-arterial cerebral thrombolysis for acute ischemic stroke. Stroke 2003;34:e109-37.

17. Tan BY, Wan-Yee K, Paliwal P, Gopinathan A, Nadarajah M, Ting E, Venketasubramanian N, Seet RC, Chan BP, Teoh HL, Rathakrishnan R, Sharma VK, Yeo LL. Good Intracranial Collaterals Trump Poor ASPECTS (Alberta Stroke Program Early CT Score) for Intravenous Thrombolysis in Anterior Circulation Acute Ischemic Stroke. Stroke 2016;47:2292-8.

18. Christoforidis GA, Karakasis C, Mohammad Y, Caragine
LP, Yang M, Slivka AP. Predictors of hemorrhage following intra-arterial thrombolysis for acute ischemic stroke: the role of pial collateral formation. AJNR Am J Neuroradiol 2009;30:165-70.

19. Yeo LL, Paliwal P, Teoh HL, Seet RC, Chan BP, Ting E, Venketasubramanian N, Leow WK, Wakerley B, Kusama Y, Rathakrishnan R, Sharma VK. Assessment of intracranial collaterals on CT angiography in anterior circulation acute ischemic stroke. AJNR Am J Neuroradiol 2015;36:289-94.

20. Fishman EK, Ney DR, Heath DG, Corl FM, Horton KM, Johnson PT. Volume rendering versus maximum intensity projection in CT angiography: what works best, when, and why. Radiographics 2006;26:905-22.

Cite this article as: Wei LM, Zhu YQ, Lu HT, Zhao JG. Thin-slab maximum intensity projection of CT angiography for collateral score and clot burden score evaluation: comparison with conventional CT angiography. Quant Imaging Med Surg 2022;12(2):1163-1171. doi: 10.21037/qims-21-170 